\title{
Temporal Chimeras Produced by Hypothalamic Transplants
}

\author{
Michael A. Vogelbaum ${ }^{1}$ and Michael Menaker ${ }^{2}$ \\ 'Departments of Biology and Biomedical Engineering, and 'Department of Biology and \\ Center for Biological Timing, an NSF Science and Technology Center, University of Virginia, Charlottesville, Virginia 22901
}

\begin{abstract}
The expression of locomotor activity by golden hamsters is temporally controlled by circadian oscillators contained within the suprachiasmatic nuclei. A genetic mutation has been found that alters the freerunning period of the locomotor activity rhythm from the wild-type value of $\approx 24$ to $\approx 20 \mathrm{hr}$ in homozygous mutants. It has been shown previously that a transplant of fetal hypothalamic tissue containing the suprachiasmatic nuclei to a host rendered arrhythmic by a complete lesion of the suprachiasmatic nuclei restores rhythmicity with the freerunning period that is normally expressed by the donor genotype. We made partial lesions of the suprachiasmatic nuclei of wild-type hosts, which did not completely abolish their circadian rhythmicity, and then placed hypothalamic implants from homozygous mutant fetal donors into the lesion site. The resulting complex patterns of locomotor activity contain rhythmic components with periods of both host and donor circadian oscillators, and suggest the presence of both stimulatory and inhibitory inputs from the circadian system to the centers controlling locomotor behavior.
\end{abstract}

Circadian rhythms in the golden hamster (Mesocricetus auratus) are normally expressed with periods close to $24 \mathrm{hr}$. However, hamsters carrying a recently discovered genetic mutation express rhythms with periods of $22 \mathrm{hr}$ (heterozygotes) or $20 \mathrm{hr}$ (homozygotes) (Ralph and Menaker, 1988). It is now clear that the mammalian suprachiasmatic nuclei (SCN) of the hypothalamus contain oscillators responsible for the generation of these rhythms. Elcctrolytic lesions that completely destroy the SCN result in a loss of circadian rhythmicity of locomotor activity in most cases (Moore and Eichler, 1972; Stephan and Zucker, 1972; Rusak, 1977; Rusak and Zucker, 1979). In contrast, partial SCN lesions, which leave some fraction of the circadian system intact, may result in disrupted activity patterns that retain clear circadian components (Gerkema et al., 1990). Transplants of fetal hypothalamic tissue containing the SCN restore circadian rhythmicity to arrhythmic, completely lesioned adult hosts (Drucker-Colin et al., 1984; Sawaki et al., 1984; Kawamura and Nihonmatsu, 1985; Lehman et al., 1987; Silver et al., 1990) with a period corresponding to the genotype of the donor tissue (Ralph et al., 1990).

\footnotetext{
Received Jan. 13, 1992; revised Mar. 30, 1992; accepted Apr. 14, 1992.

Partial support for this work was provided by Air Force Office of Scientific Research grant 90-0098 to M.M. We thank Terry Page for his critical evaluation of the initial manuscript.

Correspondence should be addressed to Michael A. Vogelbaum, Ph.D., Department of Biology, Gilmer Hall, Inniversity of Virginia, Charlottesville, VA 22901 .

Copyright (C) 1992 Society for Neuroscience $0270-6474 / 92 / 123619-09 \$ 05.00 / 0$
}

The means by which the SCN communicate their circadian signals to target systems are virtually unknown, with the exception of the neuroanatomical route by which they control pineal melatonin secretion (Klein and Moore, 1979). In particular, there have not been any previous experiments specifically designed to identify the mechanisms by means of which the mammalian circadian system controls the expression of locomotor activity, although this issue has been the subject of much speculation and formal modeling (Pavlidis, 1973; Pittendrigh, 1974; Pittendrigh and Daan, 1976; Daan and Berde, 1978; Enright, 1980). There are some published data, however, that have been used to suggest that there may be several different mechanisms responsible for the circadian control of locomotor output (Davis and Menaker, 1980; Johnson et al., 1988).

To investigate how the locomotor output system would respond to concurrent circadian signals having different periods, we transplanted fetal SCN tissue from donors of one genotype into adults of a different genotype bearing partial SCN lesions. We recorded the locomotor activity of these animals for several months after transplantation. The results of these experiments provide support for the hypothesis there are at least two qualitatively different types of output from the SCN responsible for the circadian control of the expression of locomotor activity.

\section{Materials and Methods}

SCN lesions. Each host animal was anesthetized with sodium pentobarbital $(80 \mathrm{mg} / \mathrm{kg}$ ) and placed in a stereotaxic instrument (David Kopf, Tujunga, CA). A midline incision was made on the top of the head, which had previously been cleaned with soap and water and wiped with alcohol. The bone was scraped clean of soft tissue, and a craniotomy was made at the appropriate coordinates relative to bregma. A lesioning electrode (platinum-iridium wire in a stainless steel shank coated with epoxy; $3 \mathrm{~mm}$ of the wire was exposed at the tip) was lowered through the craniotomy to the base of the third ventricle, between the two SCN. A $4.0 \mathrm{~mA}$ current was passed for $2.5,5,10$, or $15 \mathrm{sec}$ to produce SCN lesions of different sizes. The electrode was left in position for about 5 min after the lesion was made, and then removed. The craniotomy was packed with Gelfoam, and the incision was closed with surgical staples.

Preparation of implant. Homozygous mutant $\left(\tau_{\mathrm{ss}}\right)$ or wild-type $\left(\tau_{\mathrm{w}}\right)$ hamsters were mated to produce litters of known genotype; $13.5-14 \mathrm{~d}$ after mating, pregnant females were deeply anesthetized with sodium pentobarbital $(250 \mathrm{mg} / \mathrm{kg})$ and fetuses were removed by cesarian section. Each fetus was decapitated and its head was placed in a Petri dish filled with a combination of $60 \%$ Basal Medium Eagle with Earle's Salts and $40 \%$ Hanks' Balanced Salt Solution (GIBCO) maintained at $36^{\circ} \mathrm{C}$. The brains were removed from the heads and placed in another Petri dish filled with the same solution. A block of tissue containing the SCN was removed by making a coronal cut just anterior to the optic chiasm, another $1 \mathrm{~mm}$ posterior to the chiasm and two sagittal cuts $1 \mathrm{~mm}$ to each side of midline. These hypothalamic blocks were transferred to a third Petri dish containing fresh medium and kept in an incubator at $36^{\circ} \mathrm{C}$ until implantation. The time from the start of preparation of implants to completion of all implantations was less than $2 \mathrm{hr}$.

Implantation. Host animals were anesthetized and placed in the ste- 
reotaxic instrument as for a lesion, and the craniotomy made during lesioning was reopened using the tip of a scalpel. One or two donor SCN were drawn into a $5 \mu$ l Wiretrol capillary tube (Drummond Scientific Co., Broomall, PA) along with culture medium so that the total volume did not exceed $2 \mu \mathrm{l}$. The capillary tube and its plunger were mounted on a cannula guide and lowered through the craniotomy to the original coordinates for the SCN. The capillary tube was withdrawn with the plunger fixed in place until the implant was completely ejected. The tube and plunger were removed and the wound was closed as described previously. In some cases implants were made at the same time as the lesion (within $5 \mathrm{~min}$ after the current was passed); in others, implants were placed after about $30 \mathrm{~d}$.

Locomotor activity recording. Host hamsters were individually maintained in cages equipped with a running wheel, and with food and water ad libitum. Six cages were kept in a light-tight box, and each animal's running wheel activity was recorded remotely using the Dataquest (Data Services, Inc., Roseville, MN) computer-based data acquisition system. All animals were kept in constant darkness throughout the duration of the experiments, except when surgeries were performed. The data were collected as the number of running-wheel revolutions per $6 \mathrm{~min}$ bin. They were analyzed and plotted off line using our own software. Data were plotted using the standard actogram format on a $24 \mathrm{hr}$ time base and then replotted on a $20 \mathrm{hr}$ time base. Our technique of plotting the data on time bases corresponding to the periods of intact host ( $\approx 24 \mathrm{hr})$ and donor $(\approx 20 \mathrm{hr})$ hamsters allows us to determine by visual inspection alone whether or not host or donor rhythmicity is present in the locomotor activity record. We recorded the running wheel activity of each hamster starting before the SCN lesion and continuing for at least 3 months after transplantation.

Data analysis. $\chi^{2}$ periodogram (Sokolove and Bushell, 1978) analysis was performed on successive $7 \mathrm{~d}$ windows of the data, with the start of each window advanced by 1 d. $x^{2}$ critical values for the $99.95 \%$ confidence level were subtracted from the periodogram data, and negative values were set to zero. Each periodogram was calculated for test periods ranging from 18 to $26 \mathrm{hr}$ in $6 \mathrm{~min}$ intcrvals. Using the formula for overall confidence for a range of test periods given in Sokolove and Bushell (1978), we estimate a $96 \%$ confidence that all of the peaks in a single periodogram window are real. The periodogram data were plotted on a line printer using our own 3-D plotting algorithm with hidden lines removed.

\section{Results}

The results demonstrate clearly that the locomotor output system of hamsters can respond to more than one rhythmic input. Because after transplantation these animals contain circadian oscillators having both host and donor periods, we have called them "temporal chimeras." In all, we produced 44 temporal chimeras that expressed rhythms generated by both sets of oscillators; their patterns of locomotor activity fell into two classes. Examples of one class are shown in Figure 1. Figure $1 \mathrm{~A}$ shows the record of a $\tau_{w 1}$ host that received a partial SCN lesion and a $\tau_{\mathrm{ss}}$ fetal SCN implant on the same day (marked *). This animal's locomotor activity is shown on a double-plotted actogram with a $24 \mathrm{hr}$ time base (Fig. $1 \mathrm{~A}$, left); a section of the data is replotted on a $19.3 \mathrm{hr}$ time base (Fig. $1 A$, right). To simplify the description of these data, we will define a locomotor activity rhythm ("activity rhythm") to be a series of locomotor events, the onsets of which occur rhythmically with periods in the circadian range (about $18-30 \mathrm{hr}$ ). In Figure $1 A$, a locomotor activity rhythm with a period of $\approx 24 \mathrm{hr}$ can be seen in the middle of the 24 hr time base actogram (days 70-82). On days 83-88, however, this rhythm is replaced by one with a period close to $20 \mathrm{hr}$, as indicated by the fact that the onsets of activity during these days line up close to the vertical when the data are replotted on a $19.3 \mathrm{hr}$ time base. This period is consistent with what would be expected from the circadian output of the donor SCN. The $19.3 \mathrm{hr}$ actogram also reveals that this donor rhythm was expressed on days 60-70, before it was replaced by the $24 \mathrm{hr}$ rhythm described above. Although both host and donor circa- dian rhythms of locomotor activity are expressed in this animal, in this section of the data (days 60-88) they are not expressed simultaneously.

The records shown in Figure 1 also demonstrate that in addition to "activity rhythms" one can define two temporal zones that affect the expression of locomolor activity. One zone is $\alpha^{\prime}$, within which activity is allowed to occur, and the other is $\rho^{\prime}$, within which activity is not allowed to occur, or is severely inhibited (we have used the designations $\alpha^{\prime}$ and $\rho^{\prime}$ here to make clear that our definitions are derived from, but clearly distinct from, the original definitions of $\alpha$ and $\rho$ made by Aschoff, 1960). Surprisingly, both $\alpha^{\prime}$ and $\rho^{\prime}$ are seen when the data from temporal chimeras are plotted on $\approx 24 \mathrm{hr}$ time bases and also when they are replotted on $\approx 20 \mathrm{hr}$ time bases. Thus, the occurrence of each temporal zone is rhythmically determined by both host and donor circadian oscillators. Although this is evident in all of the records it is perhaps clearest in Figure $1 D$. Using the terminology employed by Davis and Menaker (1980), we call $\alpha^{\prime}$ a "window," and underline the distinction between the term "window rhythm" that we use to describe a rhythmically recurring region of allowed (rather than observed) activity, and the term "activity rhythm" described earlier.

Window rhythms are not always expressed with a constant amplitude. The record shown in Figure $1 B$ demonstrates that, while the majority of locomotor activity is expressed within each ( $24 \mathrm{hr}$ and $20 \mathrm{hr}$ ) window, some occurs outside of either window. In some cases, there is also transient expression of activity throughout entire $24 \mathrm{hr}$ cycles (Fig. 1C, left, days 5662 and days 96-105). In each of these examples, however, the boundaries of the windows are soon reestablished. The phase of each window rhythm appears to be fairly constant over the course of each of these records.

In many cases the activity rhythms seen within each window are not expressed with the same freerunning periods as are the windows themselves. Figure $1 D$ shows the record of a $\tau_{\mathrm{w},}$ host that received a $\tau_{\mathrm{ss}}$ implant immediately after a partial SCN lesion was made (marked *). On days $30-93$, it is clear that this animal's locomotor activity is expressed within two windows; one window with a period slightly longer than $24 \mathrm{hr}$ (Fig. 1D, left) and the other window with a period of about $19.8 \mathrm{hr}$ (Fig. $1 D$, right). When a series of locomotor events reaches the boundary of either the $\approx 24$ or the $\approx 20 \mathrm{hr}$ window, it disappears and then seems to reappear at the opposite boundary of the window. Other examples of this behavior can be seen clearly in Figure $1, B$ and $C$. One also can observe locomotor rhythms that appear to have periods longer than $20 \mathrm{hr}$ but shorter than $24 \mathrm{hr}$. Figure $1 D$ (days 54-82) shows the clearest example of activity rhythms with apparently intermediate periods. Figure 2 (left) shows some of the clusters of activity seen in Figure $1 D$ plotted on 24,22 , and $20 \mathrm{hr}$ time bases. The onsets of activity within each cluster are closest to vertical when the data are plotted on a $22 \mathrm{hr}$ time base, while the windows that define $\alpha^{\prime}$ and $\rho^{\prime}$ are seen clearly only on 20 and $24 \mathrm{hr}$ time bases. Similar clusters of intermediate period rhythms are seen in Figure $1 B$ (days 92-97 and days 110-114). These data also are replotted on 24,22 and $20 \mathrm{hr}$ time bases in Figure 2 (right).

Window rhythms were evident in 27 of the 44 chimeras that expressed both host and donor circadian rhythms. Their occurrence did not depend on the time of implantation of the donor SCN after the host SCN lesion (ANOVA, $p=0.650$ ), nor was it restricted to a particular host genotype.

A second class of chimeric behavior was shown by a smaller 
A

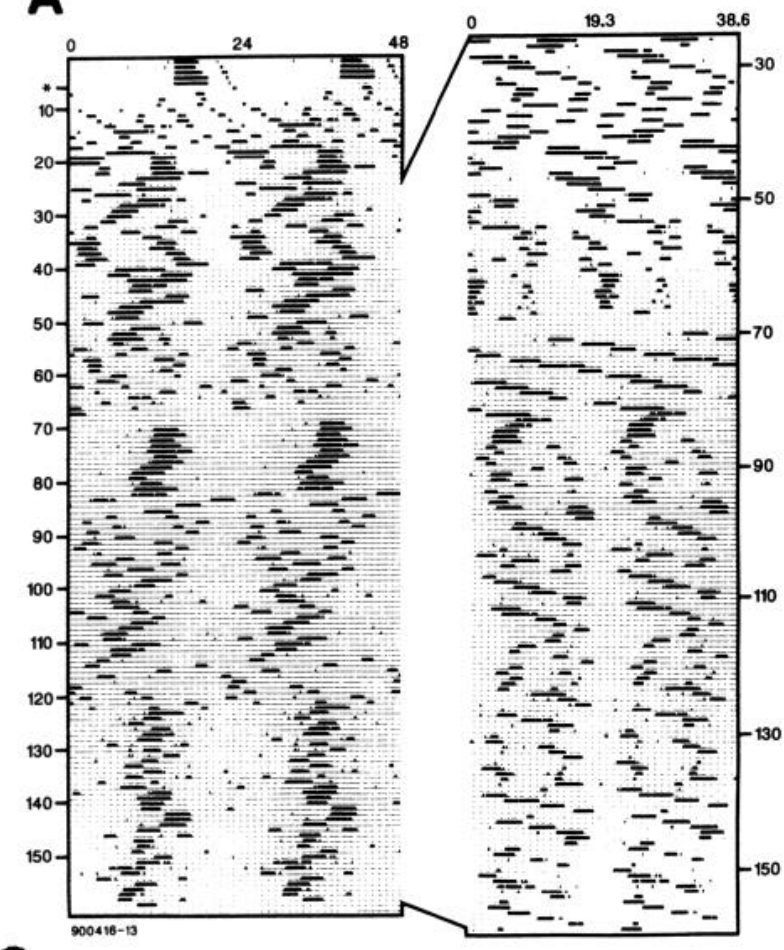

C

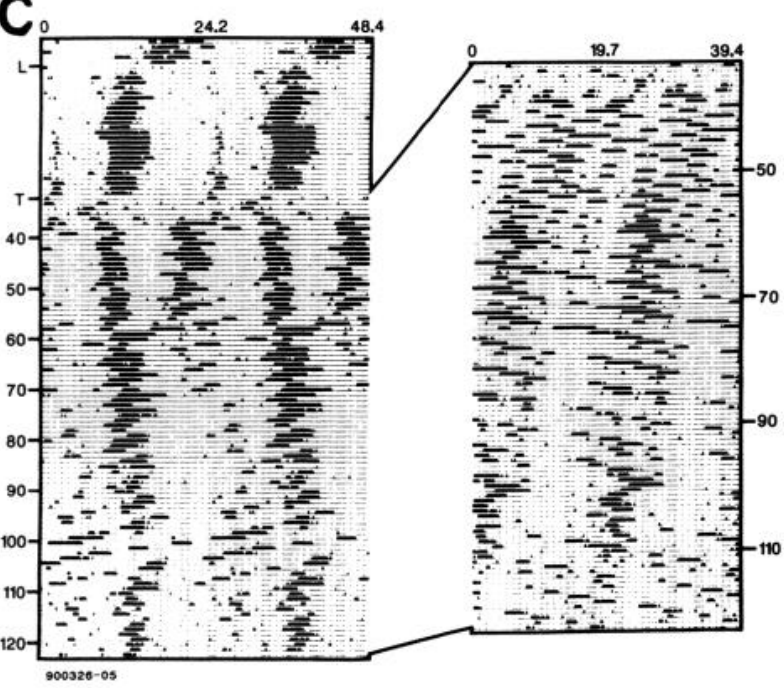

B

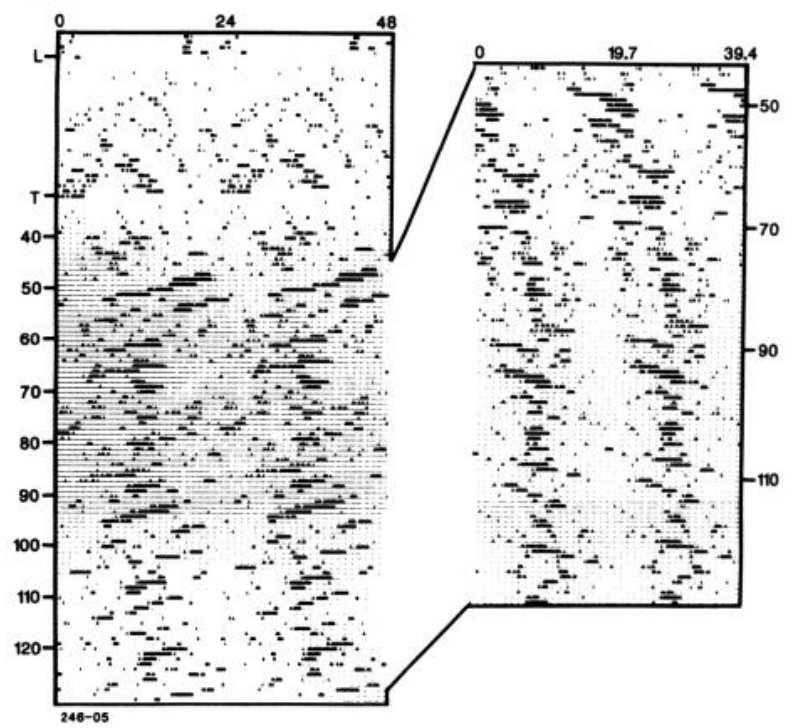

D

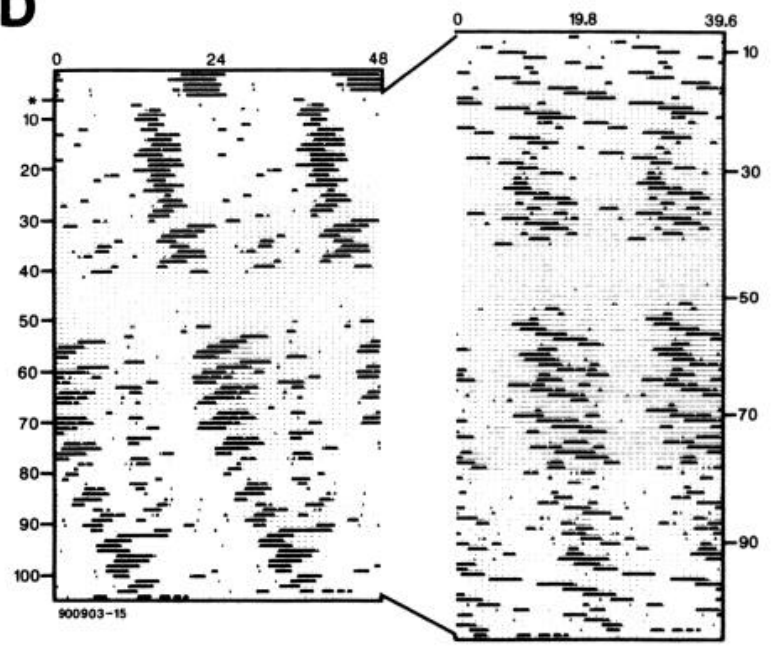

Figure 1. Wheel-running data for four temporal chimeras maintained in constant darkness. $A$ and $D$ show the records of animals in which partial SCN lesions were performed on the day marked *. These lesions were followed immediately by transplants of fetal hypothalamic blocks from homozygous mutant donors. $B$ and $C$ show the records of animals in which partial SCN lesions were made, on the day marked $L$, and then transplants were placed about $30 \mathrm{~d}$ later $(T)$. Lesion current durations for each animal whose record is shown in Figure 1 were as follows: $A$, 5 sec; $B, 5 \mathrm{sec} ; C, 5 \mathrm{sec} ; D, 2.5 \mathrm{sec}$. For each record, the left panel shows the data double-plotted on the time base that best demonstrates the period of the host's circadian rhythms, and the right panel shows a segment of the data, indicated by the lines connecting the two halves of the figure, replotted on the time base that best indicates the period of the donor rhythm. On each line of the actogram are plotted the locomotor activity data recorded during two consecutive cycles, and the second cycle's data are replotted on the line below the first day's data. Six minute time periods with more than 10 running wheel revolutions are represented by black boxes.

number of implanted animals. Figure $3 A$ shows the record of a chimera in which the expression of host donor rhythms alternates. The locomotor activity data are plotted on a $24 \mathrm{hr}$ time base (Fig. $3 A$, left) to evaluate the presence of host-derived $\approx 24$ hr rhythms. After a partial lesion of this wild-type animal's SCN was made (on the day marked "L"), a disrupted pattern of activity that retains a circadian component was seen during the next $30 \mathrm{~d}$. The period of this component became shorter fol- lowing the lesion (from $24.2 \mathrm{hr}$ before the lesion to $23.7 \mathrm{hr}$ after it). Although shortening of period is a common occurrence following partial SCN lesions, the periods always remain clearly distinguishable from those of the $\tau$ mutant hamsters (Ralph and Menaker, 1988). On the day marked "T," a homozygous mutant SCN transplant was placed into the lesion cavity. A more disrupted locomotor activity pattern was seen for the next $30 \mathrm{~d}$, but on about day 63 of the record, a new rhythmic pattern with 


\section{Timebase (Hours)}

24

22
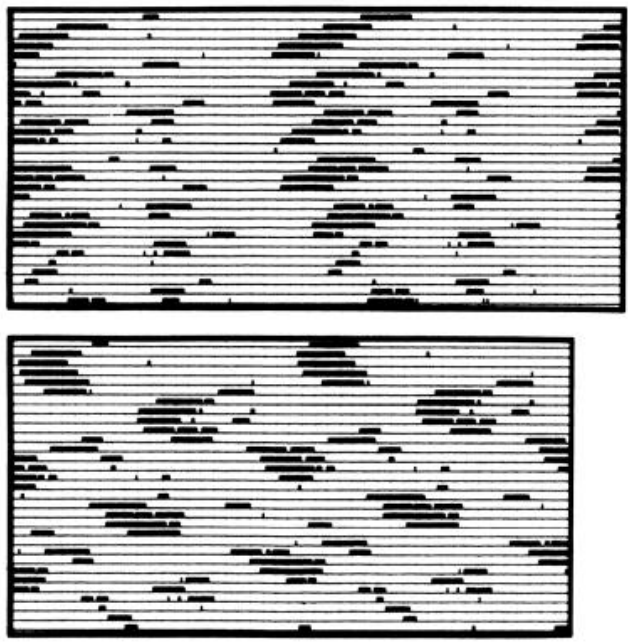

20

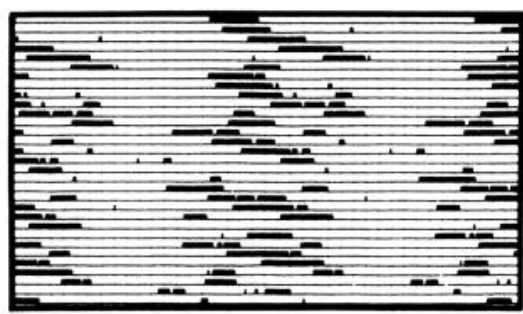

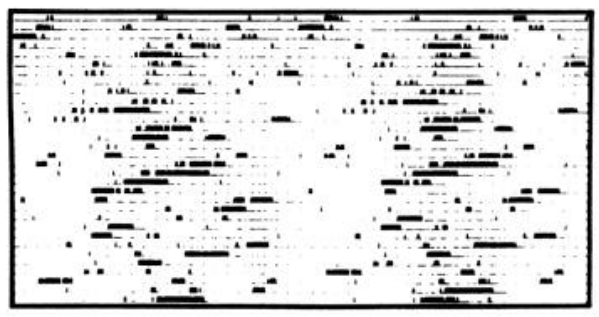
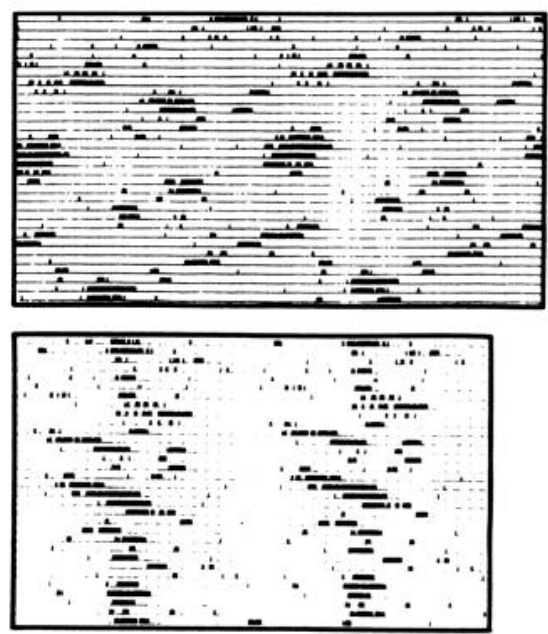

Figure 2. Segments of data from the records shown in Figure 1, B (right, days 77-108) and D (left, days 55-84) double-plotted on $24 \mathrm{hr}, 22 \mathrm{hr}$, and $20 \mathrm{hr}$ time bases.

a very short period appeared. On day 75 this rhythm was replaced by the reappearance of the $\approx 24 \mathrm{hr}$ rhythm, which in turn was interrupted by the reappearance of the shorter rhythm after about another 10 cycles. The right side of Figure $3 A$ shows a portion of the data from this record replotted on a time base corresponding to the period of the shorter rhythm. The period of this rhythm, $19.7 \mathrm{hr}$, is within the range of periods expressed by intact homozygous mutant hamsters. In this segment of the record, the $19.7 \mathrm{hr}$ activity rhythm is seen throughout, but its expression is interrupted on several occasions by the reappearance of the $\approx 24 \mathrm{hr}$ rhythm that, plotted on this time base, looks very "long." In spite of the disruptions, the $19.7 \mathrm{hr}$ rhythm maintains the same phase each time it reappears. There is no evidence that either rhythm can influence the period or the phase of the other.

The appearance of alternating host and donor rhythms does not depend upon the time of implantation. Figure $3 B$ shows the record of a hamster that received an SCN transplant immediately after a partial lesion of its own SCN had been made on the day marked by the asterisk. The left half of Figure $3 B$ shows that after about $12 \mathrm{~d}$ of arrhythmicity, a $\approx 24 \mathrm{hr}$ rhythm reappears in this record. On about day 44 , the $\approx 24 \mathrm{hr}$ rhythm is interrupted by the appearance of a shorter rhythm (cf. Fig. 3A). The $\approx 24 \mathrm{hr}$ rhythm subsequently reappears (days $48-59$ ), but is again replaced by the shorter period rhythm. Alternation of the two rhythms does not occur each time they intersect. When a segment of the data is replotted on a $19.6 \mathrm{hr}$ time base (Fig. $3 B$, right), it can be seen that the donor's rhythm is expressed more often than the host's in the second half of the record but not in the first half. This change in control over the expression of locomotor activity from the host's to the donor's circadian system is seen often and suggests an underlying plasticity in the coupling between the transplant and the locomotor output centers of the host.

Even a weakly expressed activity rhythm can alternate with one that is expressed more strongly. In the record shown in Figure $3 C, \mathrm{a} \approx 24 \mathrm{hr}$ rhythm is observable during the first $30 \mathrm{~d}$ after the transplant (days 40-58). This rhythm is then replaced by a bimodal $20 \mathrm{hr}$ rhythm, which is clearly expressed in the latter two-thirds of the record with less frequent expression of the host's $\approx 24 \mathrm{hr}$ rhythm. The paths of two oscillations with periods of 20 and $24 \mathrm{hr}$ should intersect every six $20 \mathrm{hr}$ cycles. In this record (Fig. $3 C$, right), there are a number of occasions where loss of expression of the $20 \mathrm{hr}$ locomotor activity rhythm over three to four cycles occurs at or near these intersection points, without the clear expression of a $24 \mathrm{hr}$ locomotor activity rhythm. However, this sort of interaction does not occur at all predicted crossing points. Figure $3 D$ is a running window periodogram showing the loss of the donor rhythm that occurs several times during the last one-half of the record without concomitant expression of a $24 \mathrm{hr}$ rhythm strong enough to generate more than a few small peaks. Of the 44 temporal chimeras that we have produced, 10 clearly demonstrate this alternating expression of host and donor rhythms. In the records of some animals (e.g., Fig 1 $A$ ), alternating rhythms occurred at some times and windows at others. A few of the chimeras could not be clearly classified in either the alternating or window groups.

\section{Discussion}

The data shown in Figure 1 suggest the existence of two qualitatively different rhythmic outputs from the circadian oscillators contained within the SCN. One of these outputs stimulates 
A

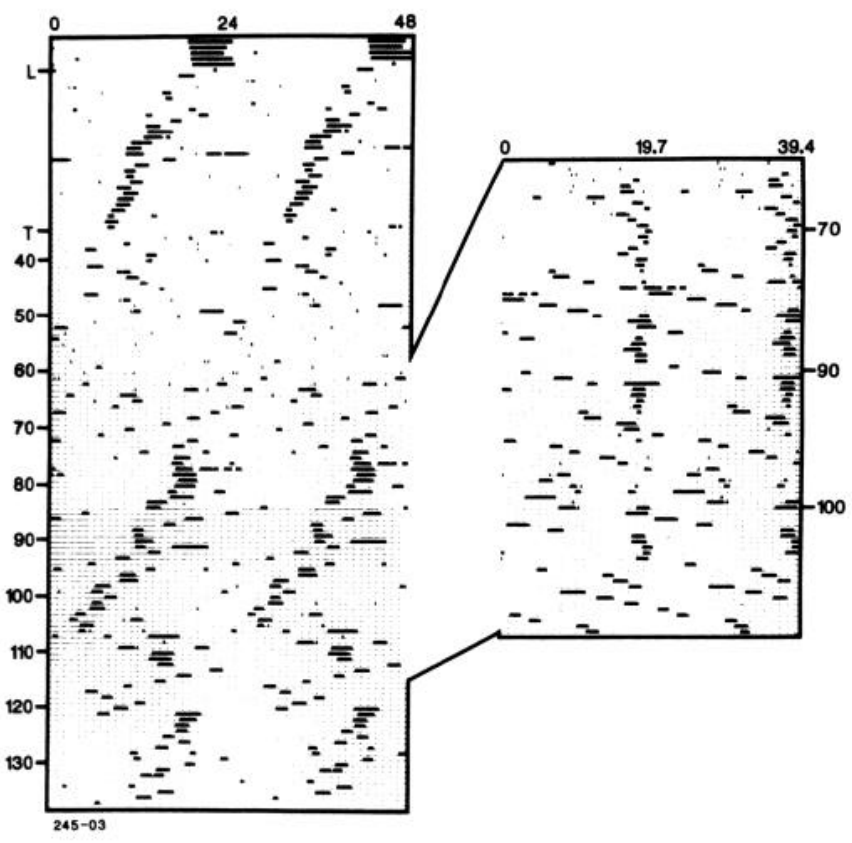

C

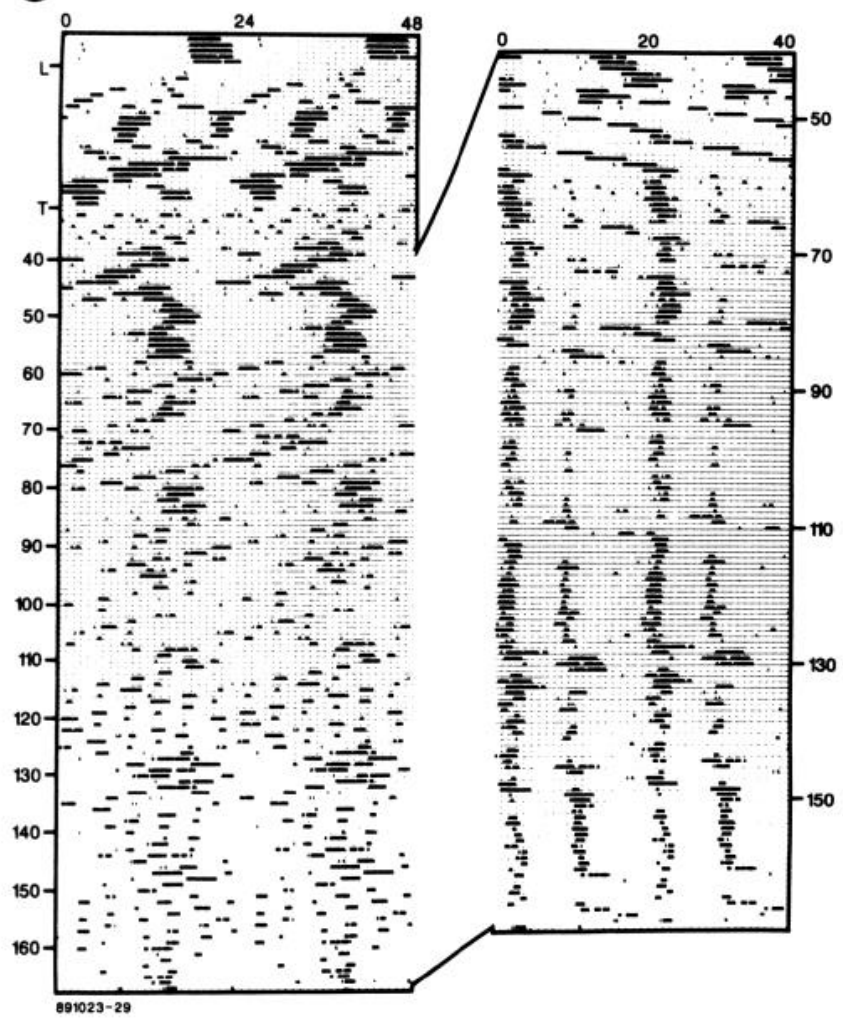

$\mathbf{B}$

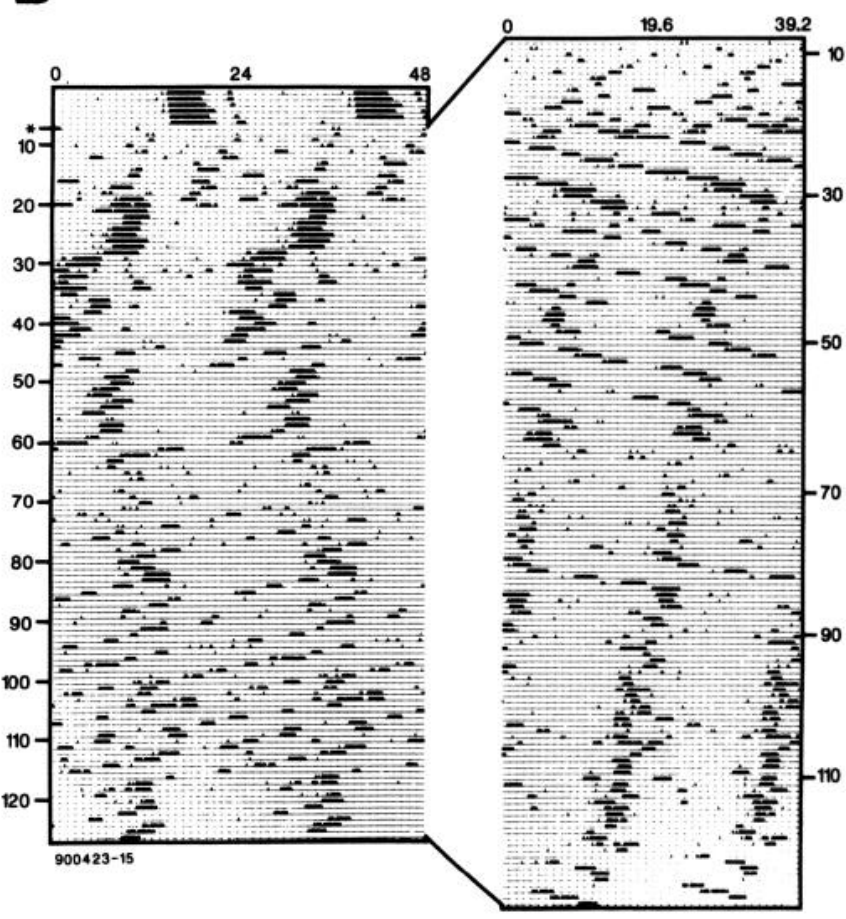

D

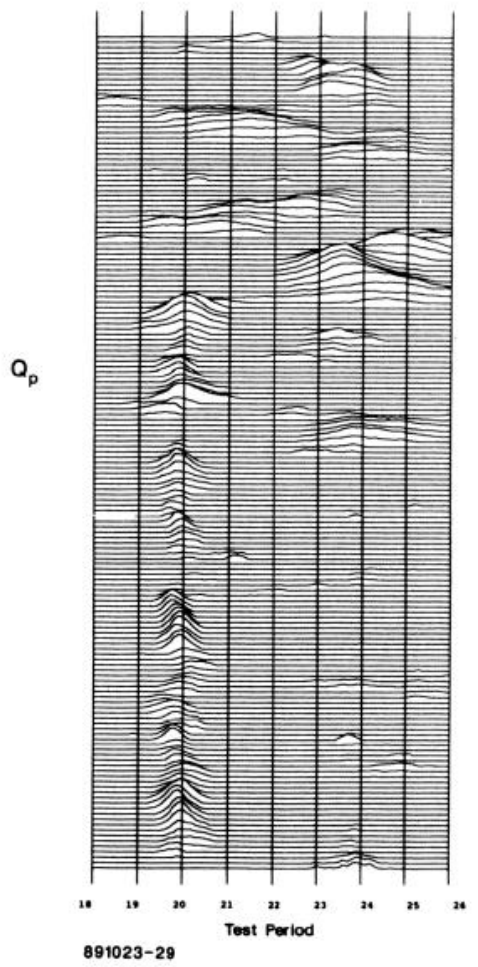

Figure 3. A, Activity record of a wild-type hamster that received a $10 \mathrm{sec}$ lesion $(L)$ and a homozygous mutant fetal SCN transplant ( $T$ ) on the days marked on the record. $B$, Activity record of a hamster that received a $10 \mathrm{sec} \mathrm{SCN}$ lesion and a homozygous mutant fetal SCN transplant on the same day $\left(^{*}\right)$. $C$. Activity record of a hamster that received a $5 \sec \mathrm{SCN}$ lesion $(L)$ and a homozygous mutant fetal SCN transplant on the day marked $T . D, 3-\mathrm{D}$ plot of the results of running window periodogram analysis of the data shown in $C$, starting after the transplant was placed. Each line represents the output of a single periodogram run on successive $7 \mathrm{~d}$ segments of the data, with the starting point of each segment advanced by $24 \mathrm{hr}$ from that of the previous segment. Upward deflections in each line represent statistically significant ( $96 \%$ confidence interval) peaks of rhythmicity for the test periods indicated on the abscissa. 


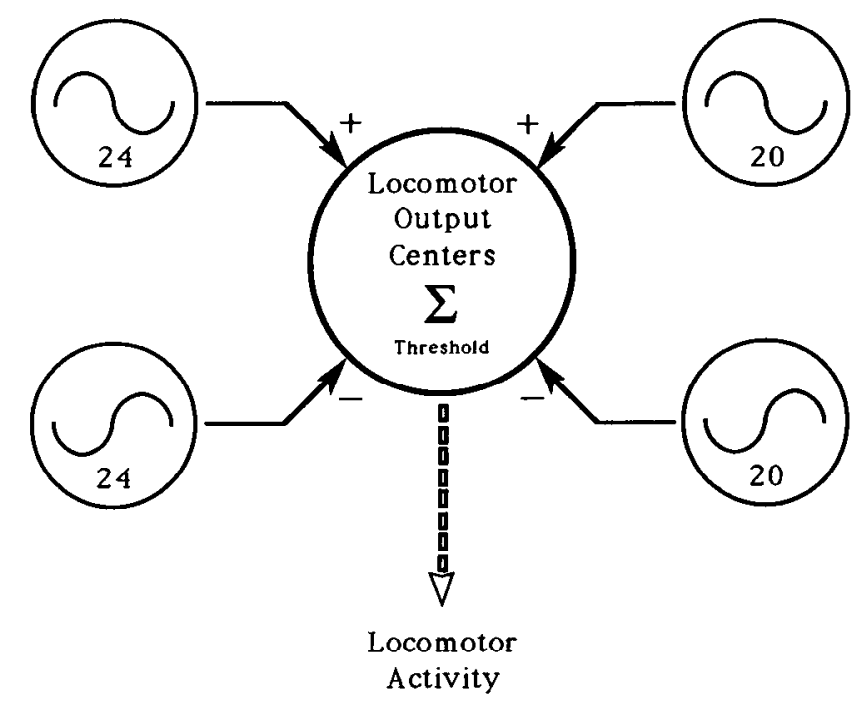

Model Equations

$$
\begin{aligned}
w(t) & =A \sin ((2 \pi * t / \tau)-\phi) \\
\mathcal{A} & \equiv \text { amplitude } \\
\tau & \equiv \text { period } \\
\phi & =\text { phase }
\end{aligned}
$$

For each rhythmic output:

$$
x(t)=\left\{\begin{array}{cc}
w(t) & w(t) \geq 0 \\
0 & w(t)<0
\end{array}\right.
$$

Figure 4. Top, A model of the control of expression of locomotor activity by host and transplanted circadian oscillators. Activity-stimulating $(+)$ and activity-suppressive $(-)$ inputs are received by the centers that drive locomotor activity. When the total level of input exceeds a threshold, these centers stimulate the expression of activity by the hamster; activity may also occur in response to stimuli not generated by the circadian system (not included in this model). Bottom, The outputs of the host and donor circadian oscillators are modeled using the equation shown. Only the positive half of the sine wave function is used in the simulations of this model. The amplitude of each excitatory and inhibitory circadian output is determined independently. The stimulatory and suppressive outputs are maintained $180^{\circ}$ out of phase for each of the simulations. The function $x(t)$ describes the output from each oscillator shown at the top. See Discussion for a description of the simulations run with this model.

the expression of locomotor activity, producing "activity rhythms" as defined earlier (which are seen within $\alpha^{\prime}$ ), and the other suppresses the expression of activity, restricting its appearance to a temporally defined window and thereby defining the boundaries of $\rho^{\prime}$. The idea that one function of a circadian output from the $\mathrm{SCN}$ is to suppress activity is novel; as far as we are aware, there are no data in the literature that directly suggest this (although many are consistent with it), and it is hard to imagine how such data could be generated without employing some "chimeric" approach such as the one we have reported here (however, see Davis and Menaker, 1980).

A detailed examination of the activity shown in each record in Figure 1 reveals the existence of the $20 \mathrm{hr}$ suppressive window rhythm crossing the $24 \mathrm{hr}$ actogram plot, as well as the $24 \mathrm{hr}$ suppressive window crossing the $20 \mathrm{hr}$ plot. On numerical grounds, we would expect the phases of 24 and $20 \mathrm{hr}$ circadian rhythms to intersect every five $24 \mathrm{hr}$ cycles. For example, an
Timebase (Hours)

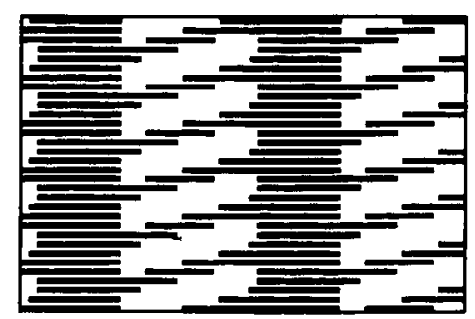

22

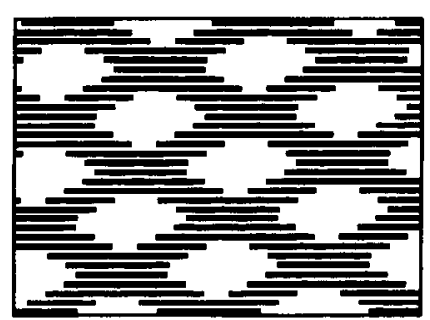

20

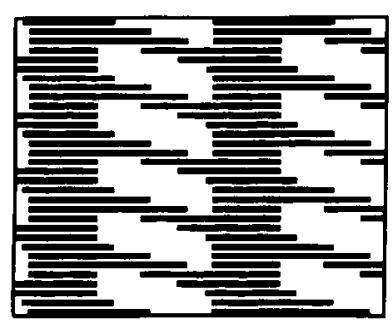

Figure 5. Activity records that result from simulations using only the activity stimulatory outputs from the host and donor circadian oscillators modeled in Figure 4. Black bars represent times during which simulated activity is driven by the locomotor output centers; the simulated activity is double-plotted on $24 \mathrm{hr}, 22 \mathrm{hr}$, and $20 \mathrm{hr}$ time base actograms in the same manner as the data shown in Figure 2. The amplitude of each stimulatory output was set equal to 50; that of each suppressive output was set equal to 0 . The threshold value was set equal to 20 .

apparently continuous $24 \mathrm{hr}$ activity rhythm in the middle of Figure $1 C$ (days 63-94) is partially interrupted about every five cycles as the $20 \mathrm{hr}$ rhythm of suppression, seen clearly in the right side of that figure, crosses the $24 \mathrm{hr}$ time base actogram. It appears that a similar suppressive window rhythm crosses through the locomotor activity rhythm seen in Figure $1 D$ (left, days 54-78). However, suppression of activity does not occur at every predicted intersection point.

We did not observe the regular or consistent appearance of phase shifts either in the activity rhythm or in the window rhythm in our data. If the output signals from each oscillator in a chimera had access to input pathways to the other oscillator, we would expect to see phase shifts as they interacted. The lack of phase shifting as the two windows intersect suggests that the suppressive window rhythm acts only to mask the appearance of locomotor activity.

On the other hand, the apparent expression of $22 \mathrm{hr}$ activity rhythms in the chimeras (Fig. 2) could indicate interaction between the host and donor oscillators. However, these apparent $22 \mathrm{hr}$ rhythms are transient and appear to change phase abruptly (see especially Fig. 2, right), and their appearance can be explained without postulating such an interaction. In Figure 4 (top) 


\section{Timebase (Hours) Data}

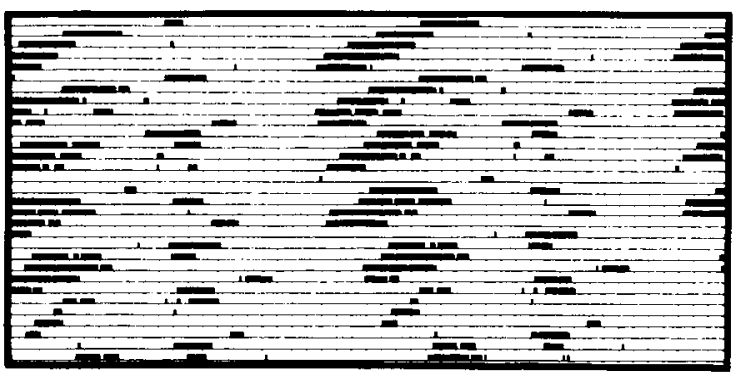

22

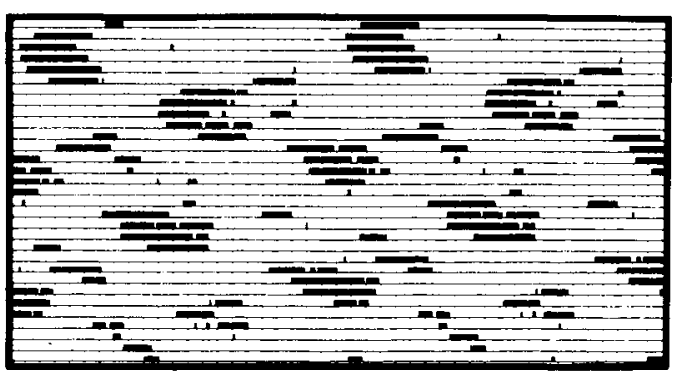

20

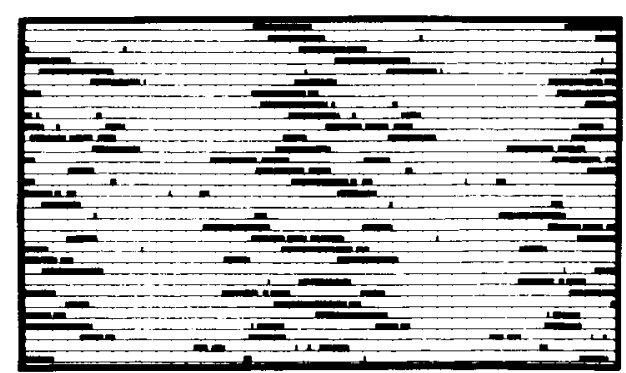

Model
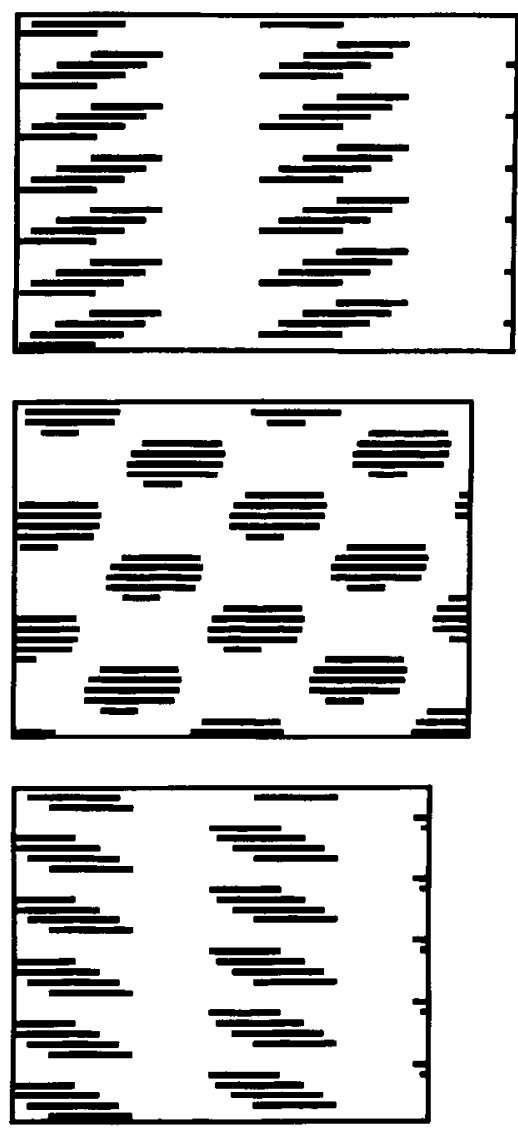

Figure 6. A comparison of the cxpcrimental data from the record shown in Figure $1 D$ (days 55-84; lefi) with the activity patterns predicted by simulations of the model shown in Figure 4 (right). For the simulated data, the amplitude of each stimulatory and suppressive output was set equal to 50 and the threshold value was set equal to 20 .

we have diagrammed a simple model that accounts for the salient features of the chimeric window rhythms. In it there is the simultaneous expression of host and donor rhythms, each of which has both an activity-stimulatory and an activity-suppressive component. The peak amplitude of the stimulating output is out of phase with that of the suppressive output. Activity stimulation increases the probability of the expression of locomotor activity while activity suppression decreases this probability. Output signals from the two $\mathrm{SCN}$ are received and instantaneously summed by the locomotor output centers. These maintain an input threshold above which locomotor activity is induced, and below which activity is not driven by the circadian system but may occur in response to other stimuli. The behavior of such a model can be visualized using a simple set of halfphase sine wave oscillators to simulate the four inputs (two from each SCN) to the locomotor output system (Fig. 4, bottom).

Using this model, it is easy to demonstrate the importance of the suppressive outputs. If only the activity-stimulating outputs from "host" and "donor" oscillators are employed both 24 and $20 \mathrm{hr}$ activity rhythms are seen simultaneously (Fig. 5). When the two activity-suppressing outputs are added, the simulated activity occurs within windows when the data are plotted on both $24 \mathrm{hr}$ and $20 \mathrm{hr}$ time base actograms (Fig. 6, right). Furthermore, when plotted on a $22 \mathrm{hr}$ time base, the simulated activity suggests the presence of components with periods close to $22 \mathrm{hr}$. A direct comparison of the locomotor activity rhythms depicted in the simulation with the experimental data from the hamster whose record is shown in Figure $1 D$ (Fig. 6, left) shows great similarity at all three time bases. Since this model was created explicitly without interaction between the "host" and "donor" oscillators, the apparent $22 \mathrm{hr}$ rhythm that emerges must be an artifact created by the masking of activity by the 24 and $20 \mathrm{hr}$ suppressive rhythms. The simulation makes clear the point that the observation of $22 \mathrm{hr}$ activity rhythms from our temporal chimeras does not indicate that the oscillators in the host and donor SCN can influence one another.

The idea of an activity-suppressive output involved in the regulation of hamster locomotor activity makes adaptive sense given the proposed multioscillator structure of the locomotor activity-generating system (Rusak, 1977). If the organization of the SCN serves to couple a population of self-sustained oscillators, then it would be crucial to suppress the expression of the stimulatory output from oscillators that are significantly out of phase with the bulk of the population, since otherwise they would stimulate activity at inappropriate times.

While this model appears to be uscful for gencrating hypotheses about the nature of the data shown in Figure 1 , in its simplest form it is not sufficient to describe the alternating pattern of rhythmicity shown in Figure 3. Nevertheless, the loss of expression of one rhythm when the other is being expressed suggests that some form of inhibition is occurring. Perhaps the alternating pattern results from competition between the host 
and donor SCN in which the output of one SCN transiently suppresses the expression of rhythmicity by the other. This hypothesis would require that as the suppressed rhythm reexpresses itself, it transiently extinguishes the expression of the other rhythm in a way analogous to the more clearly defined case of reciprocal inhibition in neuronal circuits.

A circadian organization analogous to the combined stimulatory and inhibitory circadian controls that we have found in the mammalian SCN has been described previously in the circadian system of the cockroach (Leucophaea maderae). Page $(1983 a, b)$ showed that the circadian oscillators in a transplanted optic lobe continued to cycle with a period different from that of the host despite the fact that their presence was not evident in the host's overt rhythmic behavior as long as the host's remaining optic tract was intact. However, the rhythm of the transplanted optic lobe was immediately expressed when the output pathways from the host's intact optic lobe were cut. Over time, the activity-stimulating outputs from the host's optic lobe regenerated and both host and donor circadian rhythms were expressed simultaneously (Page 1983a,b, 1984). The inhibitory output from the host optic lobe, which had suppressed the expression of the donor rhythm in intact animals, was apparently unable to regenerate its normal function completely. These results demonstrated that there is a distinct inhibitory output from the circadian oscillators in the optic lobes of the cockroach, in addition to the one that stimulates locomotor activity (Page, 1983a,b, 1984, 1988).

We do not know which cells in the SCN of hamsters are responsible for the outputs that activate or suppress locomotor behavior. There could be two subsets of circadian oscillators with one responsible for each output, or one set of oscillators with two distinct output pathways. The mode of transmission of each type of output is also unknown. There is a large and inconclusive body of literature addressing the possibility of an hormonal output mechanism (Harrington et al., 1987; Lehman et al., 1987; Aguilar-Roblero et al., 1988; Hakim and Silver, 1988; DeCoursey and Buggy, 1989; Silver et al., 1990). No data are available, however, that directly implicate either hormonal or neuronal outputs from SCN transplants. Although there are well-described projections from the intact SCN (Watts and Swanson, 1987; Watts et al., 1987), the specific functions of these projections are not known (with the exception of those innervating the pineal; Klein and Moore, 1979), and significant projectional pathways from SCN transplants have not yet been described.

There is a good deal of variability in the interactions between the circadian oscillators and the host behavioral output system in our chimeras. Window rhythms occurred in the records of most of the chimeras, but some showed the alternating pattern without expressing window rhythms. Some of the chimeras that expressed window rhythms did so only occasionally, while others showed them consistently throughout their records. Chimeras exhibiting the alternating pattern switched between expression of the host and donor rhythms every five to six cycles, as predicted, on some but not all occasions. While we are not yet able to account for this variability, we believe that it serves to emphasize the importance of using behavioral endpoints in studies involving neural transplants. Anatomical studies alone do not provide an indication of the functional significance of host-transplant interactions. Furthermore, even at the structural level they can provide only a temporal "snapshot." Since it appears that these interactions can change over long periods of time, a more dynamic assay is essential for functional assessment.

Preliminary results from the histological evaluation of the brains of our temporal chimeras indicate that grafts placed in a number of sites in or above the third ventricle can result in the successful expression of donor rhythmicity. Therefore, coupling of the circadian output signals to the host's locomotor system appears not to require close proximity between host and donor circadian oscillators.

Our data suggest that, while transplanted circadian oscillators are capable of delivering their rhythmic signals to the host's locomotor output system, they do not gain access to the host's own circadian oscillators. This result is similar to the results of previous work in which the retinal input pathways failed to make functional connections with SCN transplants (Lehman et al., 1987; Silver et al., 1990). Apparently it is difficult to reestablish input connections to the SCN. Clearly, however, the output connections of the SCN, be they neural or hormonal, are easily reformed.

\section{References}

Aguilar-Roblero RA, Morin LP, Moore RY (1988) Is fetal SCN sufficient to induce rhythm recovery when transplanted to $\mathrm{SCN}$-lesioned hamsters? Soc Neurosci Abstr 14:24.2.

Aschoff J (1960) Exogenous and endogenous components in circadian rhythms. Cold Spring Harbor Symp Quant Biol 25:11-28.

Daan S, Berde C (1978) Two coupled oscillators: simulations of the circadian pacemaker in mammalian activity rhythms. $J$ Theor Biol 70:297-313.

Davis FC, Menaker M (1980) Hamsters through time's window: temporal structure of hamster locomotor rhythmicity. Am J Physiol 239: R149-R155.

DeCoursey PJ, Buggy J (1989) Circadian rhythmicity after neural transplant to hamster third ventricle: specificity of suprachiasmatic nuclei. Brain Res 500:263-275.

Drucker-Colin R, Aguilar-Roblero A, Garcia-Hernandez F, FernandezCancino F, Rattoni F (1984) Fetal suprachiasmatic nucleus transplants: diurnal rhythm recovery of lesioned rats. Brain Res 311:353357.

Enright JT (1980) The timing of sleep and wakefulness. New York: Springer.

Gerkema MP, Groos GA, Daan S (1990) Differential elimination of circadian and ultradian rhythmicity by hypothalamic lesions in the common vole, Microtus arvalis. J Biol Rhythms 5:81-96.

Hakim H, Silver R (1988) Circadian rhythms in hamsters after SCN isolation. Soc Neurosci Abstr 14:24.14.

Harrington ME, DeCoursey PJ, Bruce D, Buggy J (1987) Circadian pacemaker transplants into lateral ventricles fail to restore locomotor rhythmicity in arrhythmic hamsters. Soc Neurosci Abstr 13:65.9.

Johnson RF, Moore RY, Morin LP (1988) Running wheel activity in hamsters with hypothalamic damage. Physiol Behav 43:755-763.

Kawamura H, Nihonmatsu I (1985) The suprachiasmatic nucleus as the circadian rhythm generator. Immunocytochemical identification of the suprachiasmatic nucleus within the transplanted hypothalamus. In: Circadian clocks and Zeitgebers (Hiroshige T, Honma K, eds), pp 55-63. Sapporo, Japan: Hokkaido Univ Press.

Klein DC, Moore RY (1979) Pineal $N$-acetyltransferase and hydroxyindole- $O$-methyltransferase: control by the retinohypothalamic tract and the suprachiasmatic nucleus. Brain Res 174:245-262.

Lehman M, Silver R, Gladstone W, Kahn R, Gibson M, Bittman E (1987) Circadian rhythmicity restored by neural transplant. Immunocytochemical characterization of the graft and its integration with the host brain. J Neurosci 7:1626-1638.

Moore RY, Eichler V (1972) Loss of a circadian adrenal corticosterone rhythm following suprachiasmatic lesions in the rat. Brain Res 42: 201-206.

Page TL (1983a) Regeneration of the optic tracts and circadian pacemaker activity in the cockroach Leucophaea maderae. J Comp Physiol 152:231-240.

Page TL (1983b) Effects of optic-tract regeneration on internal cou- 
pling in the circadian system of the cockroach. J Comp Physiol 153: 353-363.

Page TL (1984) Neuronal organization of a circadian clock in the cockroach Leucophaea maderae. In: Photoperiodic regulation of insect and molluscan hormones (Porter R, Collins G, eds), pp 115-135. London: Pittman.

Page TL (1988) Circadian organization and the representation of circadian information in the nervous systems of invertebrates. Adv Biosci $73: 67-79$

Pavlidis T (1973) Biological oscillators: their mathematical analysis. New York: Academic.

Pittendrigh CS (1974) Circadian oscillations in cells and the circadian organization of multicellular systems. In: The neurosciences: third study program (Schmitt FO, Worden FG, eds), pp 437-458. Cambridge, MA: MIT Press.

Pittendrigh CS, Daan S (1976) A functional analysis of circadian pacemakers in nocturnal rodents. V. Pacemaker structure: a clock for all seasons. J Comp Physiol 106:333-355.

Ralph MR, Menaker M (1988) A mutation of the circadian system in golden hamsters. Science 241:1225-1227.

Ralph MR, Foster RG, Davis FC, Menaker M (1990) Transplanted suprachiasmatic nucleus determines circadian period. Science 247: 975-978.

Rusak B (1977) The role of the suprachiasmatic nuclei in the gener- ation of circadian rhythms in the golden hamster, Mesocricetus auratus. J Comp Physiol 118:145-164.

Rusak B, Zucker I (1979) Neural regulation of circadian rhythms. Physiol Rev 59:449-526.

Sawaki Y, Nihonmatsu I, Kawamura H (1984) Transplantation of the neonatal suprachiasmatic nuclei into rats with complete bilateral suprachiasmatic lesions. Neurosci Res 1:67-72.

Silver R, Lehman MN, Gibson M, Gladstone WR, Bittman EL (1990) Dispersed cell suspensions of fetal SCN restore circadian rhythmicity in SCN-lesioned adult hamsters. Brain Res 525:45-58.

Sokolove PG, Bushell WN (1978) The chi square periodogram: its utility for analysis of circadian rhythms. J Theor Biol 72:131-160.

Stephan F, Zucker I (1972) Circadian rhythms in drinking behavior and locomotor activity of rats are eliminated by hypothalamic lesions. Proc Nall Acad Sci USA 69:1583-1586.

Watts AG, Swanson LW (1987) Efferent projections of the suprachiasmatic nucleus: II. Studies using retrograde transport of fluorescent dyes and simultaneous peptide immunohistochemistry in the rat. J Comp Neurol 258:230-252.

Watts AG, Swanson LW, Sanchez-Watts G (1987) Efferent projections of the suprachiasmatic nucleus: I. Studies using anterograde transport of Phaseolus vulgaris leucoagglutinin in the rat. J Comp Neurol 258: 204-229. 